\title{
EFECTOS DE DIFERENTES EMULSIFICANTES EN EL ATEMPERADO DE CHOCOLATE OSCURO
}

\author{
Effects of different emulsifiers on tempering of dark chocolates \\ Gabriela C. Chire ${ }^{1}$, Richard W. Hartel ${ }^{2}$ \\ ${ }^{1}$ Departamento de Ingeniería de Alimentos, Facultad de Industrias Alimentarias de la Universidad Nacional Agraria La Molina \\ ${ }^{2}$ Department of Food Science. University of Wisconsin-Madison, 1605 Linden Drive, Madison.
}

\section{RESUMEN}

En el presente estudio experimental se investigó el efecto de los emulsificantes: Lecitina de soya y Polirricinoleato de poliglicerol (PGPR) utilizados, en ambos casos a las concentraciones de o, y o, $3 \%$, en el atemperado del chocolate oscuro. Las muestras fueron preparadas en el laboratorio en las mismas condiciones y el efecto de la adición de los emulsificantes en el atemperado del chocolate se confirmó por medio de la curva de enfriamiento. Los resultados obtenidos indican que existe una influencia particular del grupo de PGPR a dosis de o,1\% en el grado de atemperado del chocolate oscuro ya que presentó una buena curva de enfriamiento definida por la pendiente generada con valor cero en el lapso de 2 minutos, a temperaturas entre 23,8 y $24,2{ }^{\circ} \mathrm{C}$, una vez liberada la mayor parte del calor latente prosiguió el enfriamiento debido al medio en que se encontró (agua fría) hasta que se produjo la solidificación completa del producto, respecto al emulsificante lecitina de soya, habitualmente utilizada en la industria de chocolate.

Palabras clave: Chocolate oscuro, atemperado, medidor de atemperado, curvas de enfriamiento, emulsificantes.

\section{SUMMARY}

Effects of different emulsifiers, Soy lecithin as a controller and Polyglycerol polyricinoleate (PGPR) at o,1 and o,3\% on a tempering method of dark chocolates was experimentally studied. The samples were prepared in the lab under the same conditions using a tempering method, the cooling curve represent the effect of the addition of a emulsifier about chocolate tempering behavior. The results show us certain emulsifiers of a PGPR group at o,1 $\%$ that conduct a better degree of temper of dark chocolates due to form good cooling curve with a zero slope during 2 minutes between 23,8 to $24,2^{\circ} \mathrm{C}$, where latent heat was off, finally the chocolate were solidificated, storaged and evaluated with respect to standard soy lecithin using in a chocolate production laboratory.

Key words: dark chocolate, tempering, gauge tempered, cooling curve, emulsifiers.

\section{INTRODUCCIÓN}

$\mathrm{P}$ ocos son los estudios documentados sobre los efectos de los emulsificantes en el atemperado del chocolate oscuro, por lo que este estudio preliminar busca conocer las diferentes curvas de enfriamiento producto de la adición de diversos emulsificantes tales como: Lecitina de soya (LS) estándar, usada habitualmente en la industria de procesamiento de chocolates, y los denominados polirricinoleato de poliglicerol (PGPR) que son mezclas apropiadas en diferentes proporciones de polímeros ácidos y polímeros de glicerol a concentraciones de o,1 y $0,3 \%$, utilizando un método de atemperado de chocolate oscuro ${ }^{(1)}$. El atemperado es un proceso que asegura la formación del cristal estable, aplicando un programa de temperaturas muy específico durante la producción del chocolate ${ }^{(2)}$. El grado o calidad de atemperado fue evaluado por la forma de la pendiente que presenta la curva de enfriamiento ${ }^{(3)}$ al ser graficada en una hoja de cálculo a doble escala para la muestra de chocolate, la cual fue atemperada, enfriada, registrando la velocidad de enfriamiento en función del tiempo. Los objetivos del presente estudio fueron: determinar los parámetros, como el tipo y concentración apropiados de emulsificantes para el atemperado de chocolate oscuro, para obtener una mejor curva de enfriamiento, que hacen un producto más estable en el tiempo de almacenamiento.

\section{MATERIAL Y MÉTODOS}

\section{Materias primas}

- Chocolate oscuro conteniendo $26 \%$ de grasa (p/p), sin adición de emulsificante proveniente de la empresa Guittard de Estados Unidos.

- Manteca de cacao proveniente de Ecuador.

- Emulsificantes: Lecitina de soya (LS) y diferentes tipos de PGPR fueron mezclas apropiadas en diferentes proporciones de polímeros ácidos y polí- 
meros de glicerol, provenientes de la empresa Lonza Group de Estados Unidos.

\section{Equipos}

- Tanque enchaquetado con agitador.

- Baño de agua fría: VWR Scientific, modelo 1162 , de -20 a $100{ }^{\circ} \mathrm{C}$.

- Baño de agua caliente: VWR 1130.

- Torque controlador: Master Serrodyne de o a 300 rpm, Multivolt de o a 180.

- Medidor de atemperado.

- Termocupla digital

- Computadora: Program Hyper Terminal.

\section{Métodos}

Las muestras fueron preparadas usando 1200 g de chocolate oscuro derretido entre 60 a 70 ${ }^{\circ} \mathrm{C}$ en una estufa por 60 minutos, mezcladas con manteca de cacao fundida hasta obtener el 32\% de grasa total; luego se incorporó el emulsificante a evaluar en un mezclador a $50-60{ }^{\circ} \mathrm{C}$ por dos horas, hasta obtener un producto homogéneo. Se siguió la metodología de trabajo para chocolate oscuro mostrada en la figura 1, utilizando emulsificantes al 0,1 y $0,3 \%$ porque mayores niveles dejan un gusto amargo o desagradable, especialmente a concentraciones mayores a $0,4 \%{ }^{(4)}$.

\section{Atemperado}

Se dio inicio al atemperado, que es un proceso donde el producto se sometió a ciertas temperaturas y por determinados tiempos para asegurar la formación del adecuado cristal en el chocolate ${ }^{(5)}$; esta etapa es indispensable para la obtención de tabletas de chocolate. Se trabajó de acuerdo a la siguiente metodología:

- Se continuó mezclando el chocolate completamente derretido, con el emulsificante a evaluar, entre 50 a $60^{\circ} \mathrm{C}$ y se mantuvo en agitación por una hora.

- Treinta minutos antes que termine el mezclado se encendió el motor del baño de agua fría para bajar la temperatura del chocolate, hasta llegar entre 23 y $28{ }^{\circ} \mathrm{C}$, manteniendo estable la viscosidad de la pasta; luego se incrementó la temperatura del agua fría para que la pasta de chocolate mantenga ese estado por 15 minutos y suba gradualmente hasta alcanzar de 30 a $32{ }^{\circ} \mathrm{C}$. La temperatura del agua debe estar cerca a la del chocolate con una diferencia máxima de $0,5^{\circ} \mathrm{C}$.

- El chocolate se volvió a enfriar entre 26 y $30{ }^{\circ} \mathrm{C}$ con viscosidad constante; se incrementó el calen-

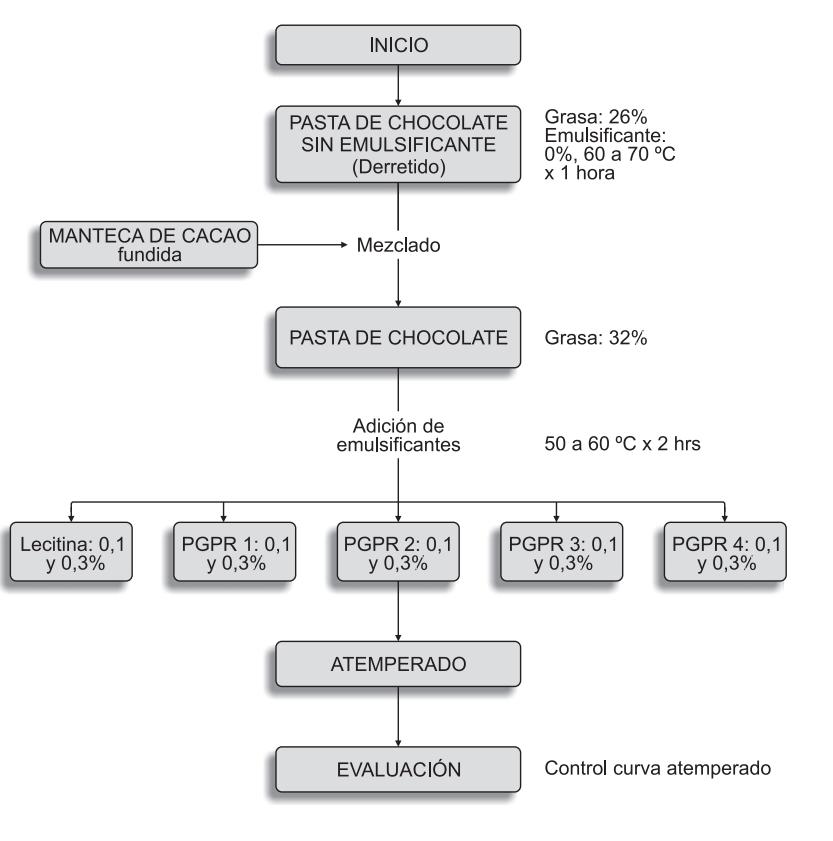

Figura 1. Diagrama de flujo

tamiento del agua hasta que la pasta de chocolate alcanzó de 32 a $34{ }^{\circ} \mathrm{C}$.

\section{Curva de enfriamiento}

Se procedió de la siguiente forma: Se usó un medidor de atemperado que consta de un cilindro de cobre donde se colocó el chocolate oscuro ${ }^{(3)}$. El registrador de temperatura tuvo dos termocuplas: una para registrar la temperatura de enfriamiento, que se colocó en el centro del cilindro de cobre -llenado con chocolate atemperado-, herméticamente cerrado, sumergido en un baño de agua fría a $0{ }^{\circ} \mathrm{C}$, y otra para registrar la temperatura del baño de agua fría. Con estos datos se obtuvo la curva de enfriamiento, que luego se graficó en una hoja de cálculo para cada tipo de variable a evaluar y para cada emulsificante. La curva se graficó en 4 minutos aproximadamente ${ }^{(5)}$.

\section{RESULTADOS Y DISCUSIÓN}

El efecto de los diferentes emulsificantes en el atemperado de chocolate oscuro fue estudiado a nivel de laboratorio, usando PGPR y como emulsificante control LS, cuyas características se presentan en la tabla 1. Una vez elaboradas las muestras, se obtuvieron los resultados descritos a continuación:

\section{Atemperado}

El atemperado dio como resultado las siguientes 
características: fácil desmoldeo de la tableta, buen "snap" (sonido fuerte al quiebre del chocolate), brillo en la tableta de chocolate y buenas características en el tiempo de vida ${ }^{(6)}$. En este proceso se controló la temperatura y el tiempo. La figura 2 muestra los cambios de temperaturas durante el proceso en el tanque, donde los chocolates fueron atemperados según el procedimiento arriba descrito. Todas las muestras pasaron por esta etapa indispensable para la elaboración del producto final.

El atemperado es un proceso que asegura la formación del cristal estable, en este caso se realizó a nivel de laboratorio en sistema cerrado. El grado o calidad de atemperado fue evaluado por la curva de enfriamiento ${ }^{(5)}$.

\section{Curva de enfriamiento}

Se conoce la curva de enfriamiento de una muestra de chocolate atemperada, por la forma que ésta presenta al graficarse la velocidad de enfriamiento en función del tiempo ${ }^{(7)}$. Una curva de enfriamiento se califica por la pendiente que tiene ${ }^{(5)}$, en el caso de una buena curva, la pendiente $(\mathrm{m})$ tiene un valor de cero (o); así mismo el tener este tipo de curva es consecuencia de un buen proceso de atemperado, lo cual ofrece ventajas en el producto final; de ahí el propósito de su estudio y la necesidad de evaluar sus efectos con los emulsificantes. A continuación, se muestran las curvas de enfriamiento para cada una de las muestras evaluadas con el medidor de atemperado de laboratorio.

Los resultados mostrados en la figura 3 nos indican que la muestra que contiene PGPR2 o,1\% presentó una buena curva de enfriamiento; esto debido a la meseta que se forma en el rango de temperaturas entre 23,8 y $24,2{ }^{\circ} \mathrm{C}$ en un lapso de 2 minutos aproximada-

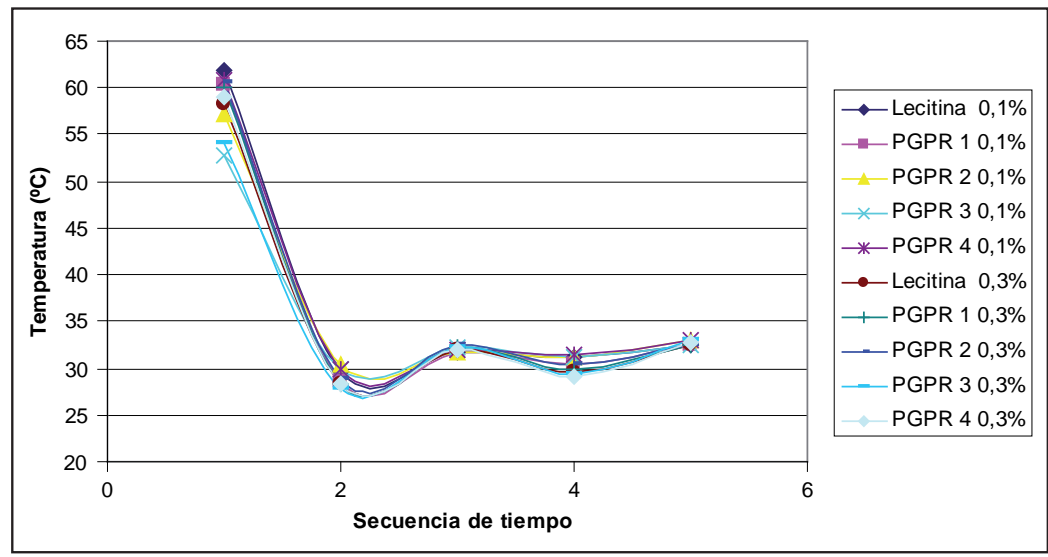

Figura 2. Secuencia de atemperado
Tabla 1. Características de los emulsificantes utilizados en el chocolate oscuro

\begin{tabular}{lcccc} 
Nombre & Dosis & Características & $\begin{array}{c}\text { Viscosidad } \\
\text { plástica }\end{array}$ & $\begin{array}{c}\text { Valor } \\
\text { mínimo }\end{array}$ \\
\hline Lecitina & 0,1 & lecitina de soya estándar & & \\
Lecitina & 0,3 & lecitina de soya estándar & & \\
PGPR 1 & 0,1 & decaglicerol & 2287 & 54.1 \\
PGPR 1 & 0,3 & decaglicerol & 2287 & 54.1 \\
PGPR 2 & 0,1 & diglicerol & 2270 & 144.6 \\
PGPR 2 & 0,3 & diglicerol & 2270 & 144.6 \\
PGPR 3 & 0,1 & emulsol SW 340 & & \\
PGPR 3 & 0,3 & emulsol SW 340 & & \\
PGPR 4 & 0,1 & palsgaard 4125 & & \\
PGPR 4 & 0,3 & palsgaard 4125 & & \\
\hline
\end{tabular}

mente (punto de inflexión), por el crecimiento activo de cristales con liberación de calor latente y generación de calor suficiente para retrasar el enfriamiento ${ }^{(3)}$. Una vez liberada la mayor parte del calor latente, prosiguió el enfriamiento debido al medio en que se encuentra el chocolate (agua fría), hasta que se produjo la solidificación completa. Las muestras conteniendo PGPR1 o,1\% y PGPR 3 o,1\% también tienden a esta forma de solidificación; sin embargo, entre éstas se aprecian diferentes curvas de enfriamiento, observables en la figura 4, esto debido a la difusividad térmica ${ }^{(8)}$ que posee cada emulsificante, ya que la pasta de chocolate es la misma en todos los casos. La muestra con PGPR3 0,1\% tiene una alta velocidad de enfriamiento: $2,07^{\circ} \mathrm{C} \mathrm{min}^{-1}$, mientras que la de las muestras PGPR1 o,1\% y PGPR2 $0,1 \%$ es de 0,93 y $1,02{ }^{\circ} \mathrm{C} \mathrm{min}^{-1}$, respectivamente. Por lo que podemos decir que la presencia de ciertos tipos de PGPR a concentraciones de o, $1 \%$ en el proceso de atemperado influyó de manera directa, positiva y rápida tal como lo reporta Hasenhuetti ${ }^{(9)}$, puesto que la curva de enfriamiento nos dio la garantía de lo sucedido como se aprecia en la figura 5 .

Existen cuatro curvas de enfriamiento ${ }^{(5)}$, con respecto al nivel de atemperado, denominadas: Sobreatemperado, cuando la pendiente $(\mathrm{m})$ tiene un valor negativo; Buen atemperado, cuando tiene valor de cero; Bajo atemperado, cuando tiene un valor positivo; y No atemperado, cuando no existe inflexión, es decir, no existe pendiente.

En el presente estudio, bajo las mismas condiciones de trabajo, se evaluó la curva de enfriamiento, obteniendo como resultados los siguientes:

- Buena curva de enfriamiento, con buen atemperado, fueron los chocolates con emulsificantes en las concentraciones 
de: PGPR1 o,1\%; PGPR2 o,1\% y PGPR3 $0,1 \%$, que poseen la apariencia y textura deseada del producto ${ }^{(10)}$.

- Sobre atemperados, fueron los chocolates con los emulsificantes: $0,1 \%$ de Lecitina; o, $1 \%$ de PGPR 4; o,3\% de Lecitina; o,3\% de PGPR 1 y o,3\% de PGPR 3, que son pegajosos, con perdida de brillo y oscurecimiento en la superficie del producto ${ }^{(10)}$.

En este caso, como las muestras fueron preparadas en las mismas condiciones, la única variante es el tipo de emulsificante, por lo que podemos concluir que algunos de los denominados polirricinoleato de poliglicerol, a bajas concentraciones, contribuyeron a una mejor curva de atemperado, cumpliéndose que las curvas de enfriamiento pueden variar dependiendo de la formulación.

\section{CONCLUSIONES}

1. Para el chocolate oscuro los parámetros promedio de atemperado fueron: $50{ }^{\circ} \mathrm{C}$ para derretir, $30{ }^{\circ} \mathrm{C}$ para enfriar, $32{ }^{\circ} \mathrm{C}$ para entibiar, $31^{\circ} \mathrm{C}$ para enfriar y finalmente $32,5^{\circ} \mathrm{C}$ para moldear.

2. La lecitina de soya estándar (LS) puede ser sustituida por emulsificantes alternativos, como algunos tipos de polirricinoleatos de poliglicerol a bajas concentraciones, dado que son favorables al proceso productivo de atemperado previo a la etapa de moldeo.

\section{AGRADECIMIENTO}

Al Dr. Richard Hartel, por su participación en la realización de la presente investigación, llevada a cabo en los laboratorios de Departamento de Ciencias de Alimentos de la Universidad de Wisconsin-Madison, USA

\section{REFERENCIAS BIBLIOGRÁFICAS}

1. Chire G, Córdova A. Mejoramiento de chocolate amargo para taza mediante el uso de licor de cacao. Ciencia e Investigación 2005; 8 (2): 87-91.

2. Rosenthal A. Textura de los Alimentos, medida y percepción. Editorial Acribia. Zaragoza, 2001.

3. Beckett S T. Fabricación y utilización industrial del cho-

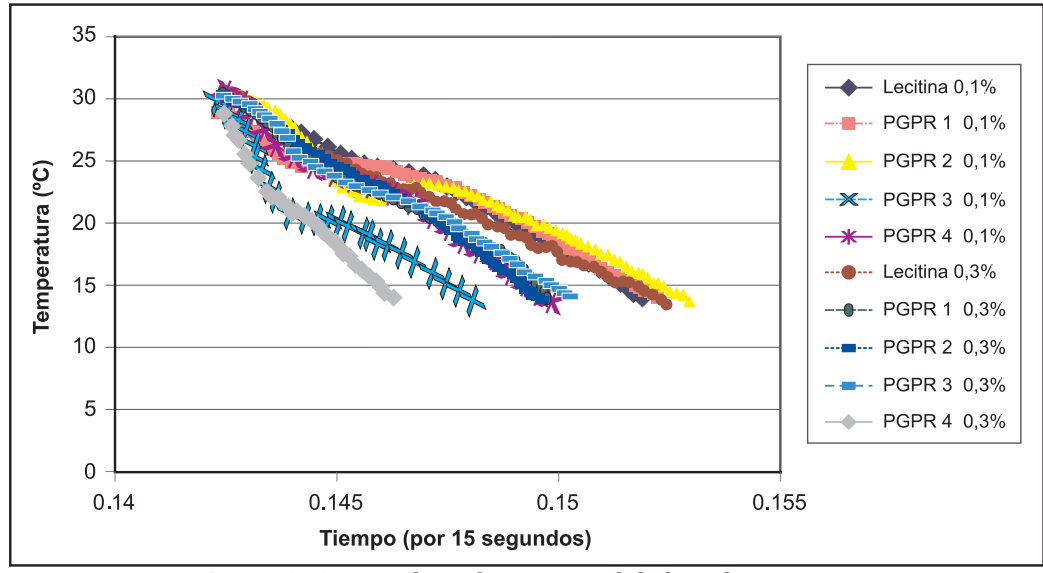

Figura 3. Curvas de enfriamiento del chocolate oscuro

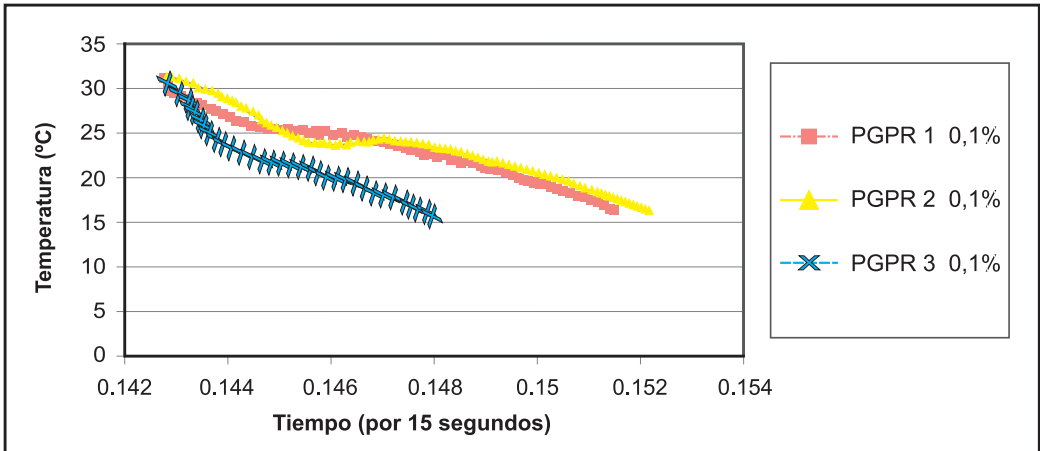

Figura 4. Curva de enfriamiento para chocolate oscuro con $0.1 \%$ de emulsificante (PGPR 1, 2 y 3)

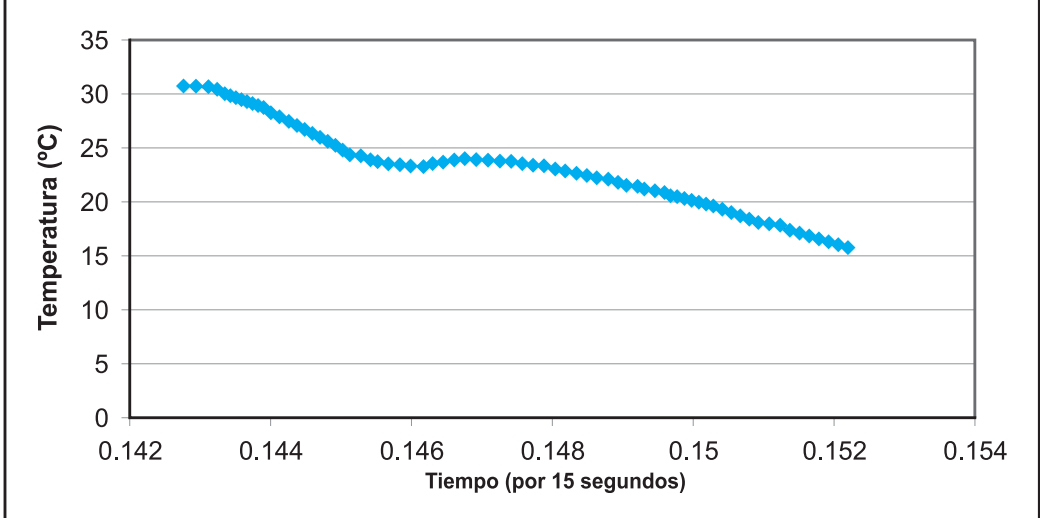

Figura 5. Curva de enfriamiento para chocolate oscuro con $0.1 \%$ PGPR 2

colate. Editorial Acribia. Zaragoza, 1994.

4. Richter M, Caetano da Silva S. Ingredientes usados na Industria de Chocolates. Brazilian Journal of Pharnaceutical Sciences 2007; 43(3): 257-369.

5. Minifie B. Chocolate, cocoa, and confectionery: Science and Technology. za Ed. Aspen Publishers. Maryland, 1999.

6. Afoakwa E, Paterson A, Fowler M, Vieira J. Modelling 
tempering behaviour of dark chocolates from varying particle size distribution and fat content using response surface methodology. Innovative Food Science and Emerging Technologies 2008; 9: 527-533.

7. Beckett S T. La Ciencia del Chocolate. Editorial Acribia. Zaragoza, 2002.

8. Lewis M J. Propiedades Físicas de los alimentos y de los sistemas de procesado. Editorial Acribia. Zaragoza, 1993.

9. Hasenhuetti G, Hartel R. Food emulsifiers and their applications. Chapman and Hall. New York, 1997.

10. Afoakwa E, Paterson A, Fowler M, Vieira J. Effect of tempering and fat crystallisation behaviour on microstructure, mechanical properties, and appearance in dark chocolate systems. Journal of Food Engineering 20o8; 89: 128-136.

Manuscrito recibido el: 22/02/2010

Aceptado para su publicación el: 15/o7/2010

\section{Correspondencia:}

Nombre: Gabriela Chire Fajardo

Dirección: Av. Los Quechuas 123, Lima 03 / Av. La Molina s/n, Lima 12 - Perú.

e-mail: gchire@lamolina.edu.pe 